\title{
The role of ultrasound in the diagnosis of a large, rapidly growing, thyroid mass
}

\author{
AD King, AT Ahuja, W King, C Metreweli
}

\begin{abstract}
Summary
The value of ultrasound in the diagnosis of a large rapidly growing thyroid mass was assessed in a study of 42 patients with a large ( $>3 \mathrm{~cm})$ rapidly growing ( $<$ two months) solitary mass. Haemorrhage into a thyroid nodule was present in 31 patients and thyroid malignancy in 11. Ultrasound of haemorrhage into a thyroid nodule revealed a large cystic mass in all 31 patients containing internal debris (22), septations (three), or a combination of both (six). The malignant causes of a large rapidly growing mass were lymphoma (two), anaplastic carcinoma (four) and metastasis (five). Ultrasound of these thyroid malignancies revealed a mass with a smooth, well-defined margin and strikingly low homogenous echogenicity in all cases. Patients with thyroid metastases had evidence of widespread metastatic disease elsewhere. Lymphoma was differentiated from anaplastic carcinoma on fine-needle aspiration cytology or surgical biopsy. Ultrasound was of value in differentiating between a benign haemorrhagic nodule and a malignant tumour. The various malignant tumours had similar appearances, however, and could not be distinguished on ultrasound.
\end{abstract}

Keywords: ultrasound, thyroid mass, malignancy

The most common indication for thyroid ultrasound is a small nodule, thyroid goiter or thyroid dysfunction. However, in our clinical practice we frequently see patients with a large rapidly growing thyroid mass. We therefore reviewed the case notes and the ultrasound findings in this group of patients with the aim of determining the cause of a rapidly enlarging mass and the value of ultrasound in diagnosis and management. The sonographic features are described and methods of differentiation are discussed.

\section{Materials and methods}

Ultrasound scans of 42 patients ( 15 male and 27 female, age range 24 to 84 years) with a history of a solitary, large $(>3 \mathrm{~cm})$, rapidly growing ( $<$ two months) thyroid mass were reviewed. Real time scans were performed with the patients supine and their necks hyperextended using a 7 and $10 \mathrm{MHZ}$ transducer. Ultrasound-guided fine needle aspiration cytology with a $21 \mathrm{G}$ needle was performed on the thyroid mass in all patients. The clinical notes were reviewed. Patients with a diagnosis of thyroid haemorrhage were only included if there were surgical correlation (9/31) or strong clinical evidence to support the diagnosis $(22 /$ 31). In the latter case this required the aspiration of altered blood (mean $18 \mathrm{ml}$ ), followed by complete collapse or a reduction in the size of the mass, with no evidence of recurrence $(9 / 22)$ or progression (13/22), on clinical follow-up for at least six months (range 6-55 months, mean 21 months) and no evidence of malignant cells on cytology. The diagnosis of malignancy was made on fine needle aspiration cytology $(7 / 11)$ or surgical biopsy (4/11).

\section{Results}

The aetiology of the rapidly enlarging thyroid mass is shown in box 1 . The size of the mass and duration of symptoms are shown in the table. All 31 haemorrhages revealed a large cystic area within a solitary nodule (21) or within a nodule of an adenomatous goiter (10). The nodules were hypo-echoic (28) or isoechoic (three), with a halo in 12 . The cystic area contained internal debris in 22, septations in three and a combination of internal debris and septations in six. There was no evidence of calcification and no cervical lymphadenopathy. No patients with the initial ultrasound diag-

\begin{tabular}{|c|c|}
\hline Aetiology of thyroid mass $(n=42)$ & \\
\hline $\begin{array}{l}\text { Benign } \\
\text { - haemorrhage into a thyroid nodule }\end{array}$ & 31 \\
\hline $\begin{array}{l}\text { Malignant } \\
\text { - metastases ( } 2 \text { sarcomas (lung and uterus), } \\
2 \text { squamous cell carcinomas from the lung, } \\
1 \text { adenocarcinoma from the breast) } \\
\text { - anaplastic carcinoma } \\
\text { non-Hodgkin's lymphoma }\end{array}$ & $\begin{array}{l}5 \\
4 \\
2\end{array}$ \\
\hline
\end{tabular}

Box 1

Table 1 Range (median) in size and duration of rapidly growing thyroid masses $(n=42)$

\begin{tabular}{lll}
\hline & $\begin{array}{l}\text { Haemorrhagic } \\
\text { nodule }\end{array}$ & $\begin{array}{l}\text { Malignant } \\
\text { tumour }\end{array}$ \\
\hline Size (cm) & $3-6(3.6)$ & $3.5-10(6.5)$ \\
Duration (days) & $2-60(30)$ & $14-60(30)$ \\
\hline
\end{tabular}




\begin{tabular}{|l|}
\hline Clinical findings in thyroid \\
malignancies ( $\boldsymbol{n}=\mathbf{1 1})$ \\
\hline Metastasis \\
- sarcoma of lung; brain metastases. \\
- sarcoma of uterus; skin and bone metastases \\
- squamous cell carcinoma of lung; bone and \\
- squamoustases. \\
- aone metastases. \\
nodenocarcinoma metastases. \\
Lymphoma of lung; skin and \\
- history of hemithyroidectomy for lymphoma \\
- Hashimoto's thyroiditis \\
Anaplastic carcinoma \\
- goiters for 10,20 and 50 years \\
\hline
\end{tabular}

Box 2

nosis of a haemorrhagic nodule were subsequently found to have malignancy.

All 11 malignant tumours had a smooth, well-defined margin; they were strongly hypoechoic and homogenous in 10/11 patients and predominantly homogenous in the eleventh patient. Necrosis was not seen but there were single foci of calcification in three patients (one metastasis and two anaplastic carcinomas). Cervical lymphadenopathy was present in four (one metastasis, one anaplastic carcinoma and two lymphomas). The clinical details are shown in box 2. Fine needle aspiration cytology was diagnostic in seven; the remaining three cases of anaplastic carcinoma and one case of lymphoma were diagnosed on biopsy.

\section{Discussion}

A rapidly enlarging thyroid mass creates much anxiety for the patient and is a frequent clinical problem. Clinical examination alone is unable to identify the nature of the mass. The differential diagnosis is usually between haemorrhage into a thyroid nodule and thyroid malignancy. The latter includes the less common tumours such as lymphoma and anaplastic carcinoma. In evaluating thyroid nodules, the role of ultrasound and fine needle aspiration cytology is well established. In differentiating benign from malignant disease, ultrasound has a sensitivity of $96 \%$, specificity of $91 \%$, and accuracy of $94 \% .^{1}$ It is therefore an ideal initial investigation.

Haemorrhage into a thyroid nodule was the commonest cause of a rapidly enlarging mass. However this study does not reflect the true incidence as many haemorrhages are aspirated in the clinic without the need for imaging and some patients who underwent ultrasound were excluded because of incomplete follow-up. On ultrasound, haemorrhage into a nodule appeared as a predominately cystic lesion with internal debris and/or septae (figure 1). These appearances have previously been described ${ }^{2,3}$ but only a few studies have included pathological correlation ${ }^{4,5}$ or long-term clinical followup. ${ }^{6}$
No malignant tumours in this study contained cystic areas. However, cystic change may occur in some malignant tumours, such as papillary carcinoma. Although we found no case of papillary carcinoma presenting as a rapidly enlarging mass, this diagnosis should be suspected if the cystic mass contains punctate calcification ${ }^{7}$ or fixed internal solid areas, ${ }^{8}$ or if there is cervical lymphadenopathy. The nodes frequently are echogenic (compared to adjacent muscle) and show punctate calcification. ${ }^{9}$ In the absence of the above features, a predominantly cystic mass with internal debris, and aspiration of altered blood (with no evidence of malignant cells on cytology) can be treated as a benign haemorrhagic nodule and followed-up.

We have shown that the malignant causes of a rapidly enlarging mass include metastases, as well as anaplastic carcinoma and lymphoma. On ultrasound these three malignant tumours revealed a sharply defined mass of surprisingly uniform homogenous echo pattern for their large size and strikingly low echogenicity (figures $2-4$ ). The extremely low echogenicity should not be mistaken for a cyst, ${ }^{10,11}$ and the sharply defined margin should not be mistaken for a sign that the tumour is benign. Previous studies have shown that hypo-echoic lesions are present in $85 \%$ of anaplastic carcinomas ${ }^{4}$ and $100 \%$ of lymphomas. ${ }^{10}$ The more com-

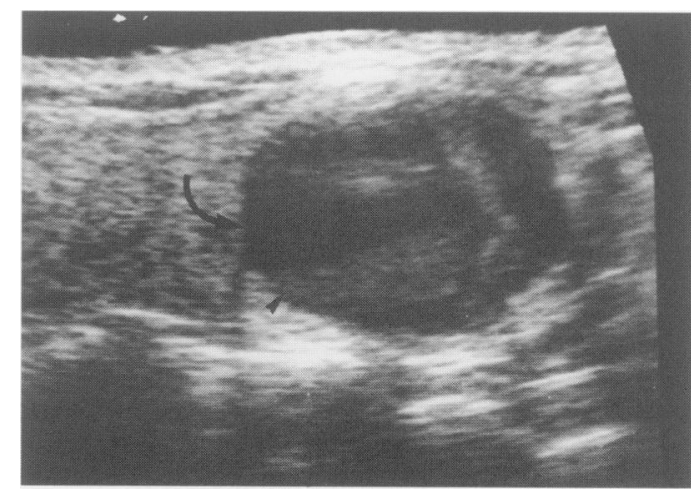

Figure 1 Longitudinal scan of the thyroid showing a haemorrhagic nodule with cystic change (curved arrow) with internal debris (arrow head) and septation (double curved arrows)

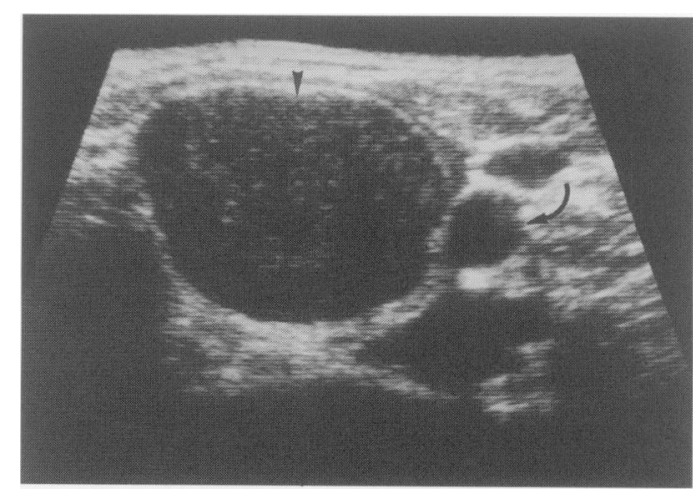

Figure 2 Transverse scan of the thyroid showing a solid, well-defined, very hypo-echoic metastatic sarcoma (arrow head). Curved arrow identifies the common carotid artery 


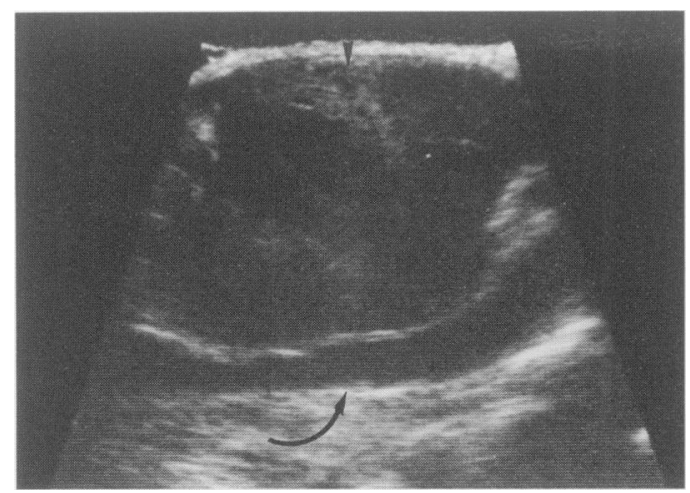

Figure 3 Longitudinal scan of the thyroid showing a solid, well-defined, very hypo-echoic anaplastic carcinoma (arrow head). Note the patent common carotid artery posteriorly (curved arrow)

mon thyroid malignancies such as papillary and medullary carcinoma also cause hypoechoic nodules on ultrasound, however these tumours usually present as cervical nodes or a small, slowly growing, nodule.

Anaplastic carcinoma, lymphoma and thyroid metastases can have similar ultrasound appearances. However, the initial diagnosis may be suggested before biopsy on the basis of clinical history. Patients with thyroid metastases usually have evidence of a primary

1 Takashima S, Fukuda H, Kobayashi T. Thyroid nodules: clinical effect of ultrasound-guided fine needle aspiration biopsy. $\mathcal{F}$ Clin Ultrasound 1994; 22: 535-42.

2 Sackler JP, Passalaqua AM, Blum M, Amorocho L. A spectrum of diseases of the thyroid gland as imaged by gray scale water bath sonography. Radiology 1977; 125: 467-72.

3 Simeone JF, Daniels GH, Mueller PR, et al. High-resolution real-time sonography of the thyroid. Radiology 1982; 145: $431-5$.

4 Solbiati L, Volterrani L, Rizzatto G, et al. The thyroid gland with low uptake lesions: evaluation by ultrasound. Radiology 1985; 155: $187-91$.

5 Scheible W, Leopold GR, Woo VL, Gosink BB. High resolution real-time ultrasonography of thyroid nodules. Radiology 1979; 133: 413-7.

6 Chang TC, Hung CT. Ultrasonographic findings in relation of ease of aspiration and fluid characteristics in thyroid cyst. f Formosan Med Assoc 1990; 89: 350-5.

7 Hatabu H, Kasagi K, Yamamoto K, et al Cystic papillary carcinoma of the thyroid gland: a new sonographic sign. Clin Radiol 1991; 43: $121-4$

8 Solbiati L, Cioffi V, Ballarati E, Ultrasonography of the neck. Radiol Clin North Am 1992; 30: 941 - 54 .

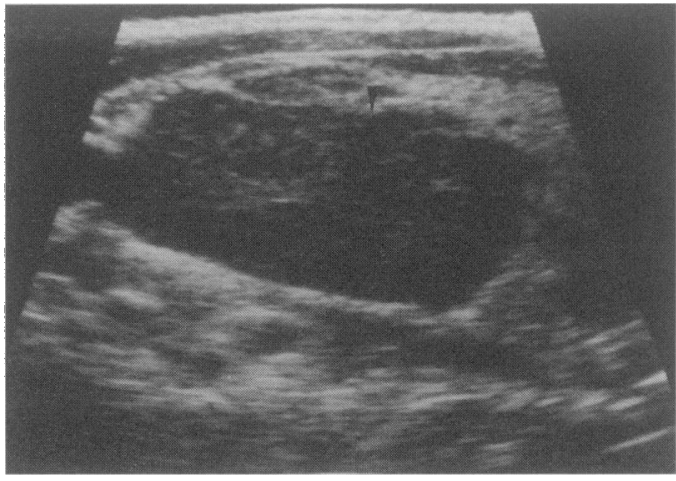

Figure 4 Longitudinal scan of the thyroid showing a solid, well-defined, very hypo-echoic primary lymphoma (arrow head)

tumour and metastatic disease elsewhere. ${ }^{12}$ Differentiation between the primary tumours is more difficult but lymphoma often occurs in patients with Hashimoto's thyroiditis, ${ }^{11,13}$ and anaplastic carcinoma may arise in a longstanding goiter. ${ }^{14}$ Fine-needle aspiration cytology is usually sufficient for the diagnosis of metastases. Surgical biopsy may be required for diagnosis of primary tumours, especially lymphoma, where specific typing is required. ${ }^{15}$

9 Ahuja A, Chow L, Mok CO, King W, Metreweli C. Metastatic cervical lymph nodes in papillary carcinoma of the thyroid. Ultrasound and histological correlation. Clin Radiol 1995; 50: 229-31.

10 Takashima S, Morimoto S, Ikezoe J, et al Primary thyroid lymphoma: comparison of CT and US assessment. Radiology 1989; 171: 439-43.

11 Matsuzuka F, Miyauchi A, Katayama S, et al Clinical aspects of primary thyroid lymphoma. Thyroid 1993; 3: $93-$ 9.

12 Ahuja AT, King W, Metreweli C. Role of ultrasonography in thyroid metastases. Clin Radiol 1994; 49: 627-9.

13 Ansocombe AM, Wright DH. Primary malignant lymphoma of the thyroid - a tumour of mucosa-associated lymphoid tissue: review of seventy-six cases. Histopathology 1985; 9: $81-97$.

14 Degroot LJ, Sridama V. Thyroid neoplasia, In: Degroot LJ, ed, Endocrinology, 2nd edn. Philadelphia: WB Saunders, 1989; pp 758-76.

15 Takashima S, Nomura N, Noguchi Y, Matsuzuka F, Inoue T. Primary thyroid lymphoma: evaluation with US, CT, and MRI. 7 Comput Assist Tomogr 1995; 19: 282-8. 